\title{
Diagnostic and prognostic impact of serum HE4 detection in endometrial carcinoma patients
}

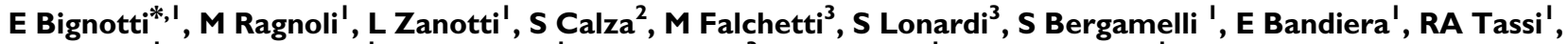 \\ C Romani', P Todeschini', FE Odicino', F Facchetti ${ }^{3}$, S Pecorelli' and A Ravaggi' \\ l'Angelo Nocivelli' Institute of Molecular Medicine, Department of Obstetrics and Gynecology, Division of Gynecologic Oncology, University of Brescia, Viale \\ Europa II, 25 123, Brescia, Italy; ${ }^{2}$ Section of Medical Statistics and Biometry, Department of Biomedical Sciences and Biotechnology, University of \\ Brescia, Viale Europa II, 25 I23, Brescia, Italy; ${ }^{3}$ Department of Pathology, University of Brescia, Viale Europa II, 25 I 23, Brescia, Italy
}

BACKGROUND: To date, no good marker for screening or disease monitoring of endometrial cancer (EC) is available. The aims of this study were to investigate HE4 gene, protein expression and serum HE4 (sHE4) levels in a panel of ECs and normal endometria (NEs) and to correlate sHE4 with patient clinicopathological characteristics and prognosis.

METHODS: Using quantitative real-time PCR we tested 46 ECs and 20 NEs for HE4 gene expression. Protein expression was analysed by immunohistochemistry on tissue microarrays in I53 ECs and 33 NEs. Pre-operative serum samples from I38 EC and 76 NE patients were analysed with HE4-EIA assay. Association between sHE4 and patient clinicopathological characteristics or outcome was evaluated.

RESULTS: Protein and HE4 gene were significantly upregulated in EC tissues and sera, compared with controls. High sHE4 levels were significantly associated with worse EC clinical characteristics. By univariate survival analysis, high sHE4 levels significantly correlated with decreased overall survival, progression-free survival and disease-free survival, retaining their independent prognostic value on the poorly differentiated EC cohort.

CONCLUSION: We demonstrate, for the first time, that high sHE4 levels correlates with an aggressive EC phenotype and may constitute an independent prognostic factor for poorly differentiated-ECs. Determination of sHE4 could be clinically useful in identifying high-risk EC patients for a more aggressive adjuvant therapy.

British Journal of Cancer (201 I) 1 04, 1418-1425. doi: I0.1038/bjc.201 I.109 www.bjcancer.com

Published online 5 April 2011

(C) 20II Cancer Research UK

Keywords: serum marker; endometrial carcinoma; HE4; diagnosis; prognosis

Endometrial carcinoma (EC) is the most common gynaecological malignancy in the Western world. (Sherman, 2000; Amant et al, 2005). Most EC patients have oestrogen-related tumours, well-differentiated, endometrioid in histology and, consequently, with good prognosis (Type I ECs). In contrast, Type II ECs include non-oestrogen-related poorly differentiated endometrioid, serous papillary and clear-cells ECs, which being aggressive and metastatic at presentation, often recur despite aggressive clinical interventions (Bokhman, 1983). Unfortunately, to date, no good marker for EC screening, early diagnosis or disease monitoring is available. In this regard, CA125 serum determination is often used in clinical practice to monitor EC patients (Duk et al, 1986). However, this marker appears to have limited utility in analysing the effects of adjuvant therapy or in the prediction of tumour recurrence. Endometrial carcinoma conventional prognostic factors are tumour grade, International Federation of Gynecology and Obstetrics (FIGO) staging and histological type (Bokhman, 1983; Cirisano et al, 1999, 2000; Creasman et al, 2001; Gemer et al, 2004), but they are insufficient to identify patients with poor

*Correspondence: Dr E Bignotti; E-mail: bignottieliana@yahoo.it Received 4 January 20I I; revised 4 March 201 I; accepted 8 March 20I I; published online 5 April 2011 prognosis. The recent progress in molecular biology has identified novel biological markers (Kohler et al, 1996; Risinger et al, 1998; Maxwell et al, 2001; Morrison et al, 2006) representing important alternative indicators of patients with biologically aggressive, highrisk tumours, which may benefit from adjuvant therapies to improve outcome (Greven et al, 2006; Randall et al, 2006).

Human epididymis protein HE4 (WFDC2) was first identified by Kirchhoff et al (1991) and specifically localised to the epithelial cells of the epididymal duct. More recently, HE4 expression has been reported in a number of normal human tissues outside of the male reproductive system, as well as in various types of human carcinomas, including EC (Bingle et al, 2002; Galgano et al, 2006). A recent paper of Moore et al (2008) demonstrated that HE4 preoperative serum levels discriminate EC patients from healthy postmenopausal women, suggesting its promising value as diagnostic serological marker to be used alone or in combination.

In the present report, we have analysed HE4 gene expression levels by quantitative real-time PCR, whereas protein expression was tested by immunohistochemistry on tissue microarrays in a large cohort of EC patients. Furthermore, with the aim to confirm HE4 potential utility in EC diagnosis, using a commercially available ELISA assay, we have measured preoperative HE4 serum levels in a large cohort of EC patients and in healthy controls. In 
addition, we have compared HE4 serum levels with those of CA125, the marker more often used in clinical practice. Finally, we have investigated the correlation between HE4 serum levels and either clinical factors or survival end points to determine its potential prognostic significance. The univariate and multivariate survival analyses were performed both on the entire cohort of EC patients and on the poorly differentiated EC subgroup.

\section{MATERIALS AND METHODS}

\section{Human tissue and serum samples and patient characteristics}

EC tissue samples were obtained from 153 patients treated at the Division of Gynecologic Oncology of the University of Brescia, Italy between September 2003 and July 2009. Moreover, samples of normal endometria (NE) were collected from 33 age-matched patients, undergoing surgery for benign pathologies. Preoperative serum samples from 138 patients with EC and from 76 controls (postmenopausal women without gynaecological pathologies) were collected. The study was approved by the Institutional Review Board and written informed consent was obtained from all the patients enrolled. All pathology specimens were reviewed in our institution and histological classification was performed according to the WHO criteria, whereas pathological stage was determined in accordance with the FIGO guidelines. Tumour tissues were obtained from women undergoing total abdominal hysterectomy, bilateral salpingo-oophorectomy and peritoneal washings for cytology. Lymph node sampling or dissection was predominantly performed in patients with tumours characterised by deep myometrial invasion and/or high-grade or aggressive histology. The presence of serious concomitant diseases, obesity and advanced age were contraindications to full-surgical staging. None of the patients had received preoperative chemotherapy or radiation. Age, histological type, stage, grade, additional disease (i.e., diabetes, obesity and blood pressure) and treatment information were recorded in all cases. A group of 134 patients was selected for survival analysis. Patients were followed up from the date of surgery until death or the last observation (median follow-up, 33.1 months, range 0.9-74.4 months). At the time of the last follow-up, 107 patients (79.9\%) were alive without evidence of disease, 8 patients $(6.0 \%)$ were alive with disease, 18 patients (13.4\%) were dead from disease and 1 died from other causes. For subgroup survival analysis, 54 patients harbouring poorly differentiated ECs were considered.

\section{Total RNA extraction and quantitative real-time PCR}

Tissue-sharp dissection, liquid nitrogen freezing and epithelial purity checking of EC and NE samples were performed as previously reported (Bignotti et al, 2006), as well as total RNA extraction and reverse transcription. Quantitative real-time PCR was performed in triplicate as previously reported (Bignotti et al, 2006), using the following Assay on Demand (Applied Biosystems, Foster City, CA, USA): Hs00899484_m1 (WFDC2). Normal and EC samples were analysed at the same time to avoid batch effects. Data were normalised using glyceraldehyde-3-phosphate dehydrogenase as internal control.

\section{Tissue microarrays and immunohistochemistry}

Tissue microarray blocks (TMAs) were created from 153 formalinfixed, paraffin-embedded EC and $33 \mathrm{NE}$ tissues collected from the Department of Surgical Pathology of the University of Brescia, Italy. Tissue microarray blocks were constructed using an automated tissue microarrayer (TMA Master, 3DHistech, Budapest, Hungary). Representative areas were chosen for sampling from haematoxylin and eosin (H\&E) stained sections of selected
$\mathrm{NE}$ and EC cases of different histological subtypes. Four 0.6-mm cores have been collected from different areas of each tumour block in order to overcome tumour heterogeneity and the possible loss of tissue because of cutting. Different TMAs were created for low and advanced tumour grade, and normal tissue was included in all TMAs to be used as control. Sections ( $4 \mu \mathrm{m}$ thick) were cut from TMAs and H\&E staining were used for confirmation of tumour tissue. Tissue microarray sections were subjected to antigen retrieval $(15 \mathrm{~min}$ in microwave oven at $750 \mathrm{~W}$ in sodium citrate buffer, $\mathrm{pH} 7.0$ ), before application of the rabbit polyclonal antibody to HE4 (1:40 dilution; Covance, Dedham, MA, USA). The antibody was revealed with NovoLink polymer (Novocastra Laboratories, Newcastle upon Tyne, UK), followed by diaminobenzidine as chromogen; hematoxylin was used for counterstaining. Immunoreactivity was evaluated by four independent observers; cytoplasmic staining was graded for intensity (0-negative, 1-weak, 2-moderate and 3-strong) and the percentage of positive cells was scored as $0(0 \%), 1(1-10 \%), 2(11-50 \%)$ and $3(51-100 \%)$. A single scale with scores $0-9$ was obtained by multiplying the intensity and the percentage staining score, and a total score was calculated by grouping score 0 in total score $0,1-3$ in total score 1, 4 and 6 in total score 2 and 9 in total score 3. Digital images were resized by using Adobe Photoshop (Element version) in order to have homogeneous cores (about $90 \%$ of the full core is represented).

\section{HE4 Immunoassay}

All serum samples were collected, before any patient treatment, frozen in liquid nitrogen within $2 \mathrm{~h}$ of blood drawing and stored at $-80^{\circ} \mathrm{C}$. Serum HE4 levels were measured using HE4 EIA kit (Fujirebio Diagnostics, Inc., Goteborg, Sweden), following manufacturer's instruction. Normal and EC samples were analysed in duplicate at the same time to avoid batch effects.

\section{CA125 serum level measurements}

Serum CA125 values were determined by the clinical laboratory at the Spedali Civili di Brescia, Italy using the Architect CA125 II chemiluminescent two step immunoassay kit (Abbott Diagnostics, Abbott Park, IL, USA), following the manufacturer's protocol. Normal and EC samples were analysed at the same time to avoid batch effects.

\section{Statistical analysis}

In all the analyses, HE4 gene expression has been considered on log-scale. Differences in HE4 gene expression between ECs and NEs were evaluated with $t$-test, whereas IHC values were tested with the Wilcoxon rank sum test and Mann-Whitney $U$-test. Differences in HE4 serum levels between the groups were calculated using ANOVA. Spearman's rank correlation was used to estimate the degree of association between serum HE4 (sHE4) and CA125 values, whereas Kendall's coefficient was used to estimate the degree of concordance between IHC and ELISA data. Combination of markers was investigated comparing ROC curves, based on the method proposed by DeLong et al (1988). Different ROCs are built, based on predicted values for each sample derived from logistic models, accounting for different combinations of markers. Predicted values are computed using leave-one-out cross validation. The association between HE4 serum levels and clinicopathological parameters was investigated with ANOVA. For survival analysis, three end points (cancer relapse, cancer progression and death due to cancer) were used to calculate disease-free survival (DFS), progression-free survival (PFS) and overall survival (os), respectively. Disease-free survival was defined as the time interval between the date of surgery and the date of identification of disease recurrence, PFS was defined as the time 
interval between the date of surgery and the date of identification of progressive disease (disease not treatable with curative intent) and OS was defined as the time interval between the date of surgery and the date of death. For all three end points, the last date of follow-up was used for censored subjects. Survival models were fitted using the Cox proportional hazard models, whereas survival curves were drawn based on the Kaplan-Meier methods. The effect of HE4 serum levels on prognosis was evaluated by categorising the values in tertiles computed on the entire cohort (low; medium; high). In all the analyses, a $P$-value $<0.05$ was considered significant. All the analyses were performed using $R$ (R Development Core Team, 2010).

\section{RESULTS}

\section{HE4 gene expression by quantitative RT - PCR}

Human epididymis protein HE4 gene expression was tested by $\mathrm{qRT}-$ PCR in 46 ECs (20 poorly differentiated (G3), 17 moderately differentiated (G2) and 9 well differentiated (G1)) and 20 NEs. As shown in Figure 1, HE4 mRNA expression was significantly higher in EC compared with NE patients $(\mathrm{FC}=2.63,95 \% \mathrm{CI}: 1.78-3.88, P<0.0001)$.

\section{HE4 protein expression by immunohistochemical staining}

To analyse HE4 expression results at the protein level, immunohistochemistry for HE4 was carried out on 153 EC (53 G3, $60 \mathrm{G} 2$ and $40 \mathrm{G} 1$, including 119 endometrioid ECs and 34 nonendometrioid ECs) and 33 NE (16 during proliferative phase and 17 during secretory phase) TMAs. As shown in Table 1, a positive staining for HE4 was detected in 130 out of $153(85.0 \%)$ ECs and appeared to be moderate/strong (score $2 / 3$ ) in $59.5 \%$ of cases. Normal endometria tissues predominantly showed a weak immunoreactivity, with presence of score 1 in $57.6 \%$ of cases. Endometrial cancers showed markedly increased HE4 positivity as compared with NEs $(P=0.03)$. As represented in Figure 2, HE4 staining in EC and NE samples appeared to be cytoplasmic and restricted to the epithelial compartment, with no positivity in adjacent stromal cells.

As displayed in Table 1, all G1 cases were positive and most of them (31 out of $40,77.5 \%)$ showed a cytoplasmic staining with moderate/strong score. Most of G2 cases (53 out of $60,88.3 \%$ ) were also found positive for HE4 and showed a moderate/strong score in $65 \%$ of cases, whereas G3 tumours (32 out of $53,60.4 \%$ ) were mainly scored negative/weak. Immunostaining of HE4 was significantly greater in G1 and in G2 tumours compared with G3 ones $(P<0.0001$ and $P=0.0062$, respectively). Moreover, immunoreactivity for HE4 was significantly stronger in endometrioid ECs compared with non-endometrioid ECs $(P=0.016)$.

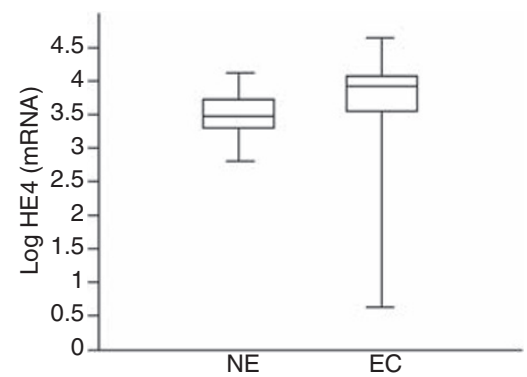

Figure I HE4 mRNA expression in endometrial carcinoma (EC) compared with normal endometrial tissues (NE). The figure shows the box plot of the relative quantification values in Log scale. As shown, HE4 mRNA expression was significantly higher in EC compared with NE patients $(P<0.000 \mathrm{I})$.

\section{Serum HE4 levels}

Serum samples were collected from 138 EC patients and 76 healthy controls and tested with HE4 ELISA. Median, mean and range values for each group are displayed in Figures $3 \mathrm{~A}$ and B. As shown, sHE4 levels were significantly higher in EC patients compared with NEs (median ECs $=83 \mathrm{pM}$, median NEs $=38 \mathrm{pM}, \quad \mathrm{FC}=2.33$, $P<0.0001,95 \%$ CI: 2.02-2.73), regardless of the FIGO stage (NEs $v s$ Stage I ECs, $P=0.004$; NEs $v s$ stage II, III or IV ECs, $P<0.0001$ ) and differentiation grade (NEs vs G1, G2 or G3 ECs, $P<0.0001)$. Moreover, sHE4 levels in G1 ECs showed a significant difference with G2 ECs (FC $=1.64, P=0.0009,95 \%$ CI: $1.22-2.20)$. The comparisons between G2 ECs and G3 ECs and between G1 ECs and G3 ECs were not significant $(P=0.240$ and $P=0.066$, respectively). In addition, sHE4 levels were higher in patients with advanced FIGO stages: I-II (mean 90) vs III-IV stages (mean $174), P=0.014$. Finally, sHE4 levels and IHC results showed a low concordance in paired tumour samples $(P=0.18, W=0.563)$.

Table I HE4 immunoreactivity in tissue microarrays of endometrial carcinomas (ECs) and normal endometria (NEs)

\begin{tabular}{cccccc}
\multicolumn{7}{c}{ HE4 protein expression } \\
\hline & $\boldsymbol{n}$ & $\begin{array}{c}\text { Score }=\mathbf{0} \\
\boldsymbol{n}(\%)\end{array}$ & $\begin{array}{c}\text { Score }=\mathbf{I} \\
\boldsymbol{n}(\%)\end{array}$ & $\begin{array}{c}\text { Score }=\mathbf{2} \\
\boldsymbol{n}(\%)\end{array}$ & $\begin{array}{c}\text { Score }=\mathbf{3} \\
\boldsymbol{n}(\%)\end{array}$ \\
\hline$E C S$ & 153 & $23(15.0)$ & $39(25.5)$ & $61(39.9)$ & $30(19.6)$ \\
GI & 40 & $0(0.0)$ & $9(22.5)$ & $16(40.0)$ & $15(37.5)$ \\
G2 & 60 & $7(11.7)$ & $14(23.3)$ & $27(45.0)$ & $12(20.0)$ \\
G3 & 53 & $16(30.2)$ & $16(30.2)$ & $18(34.0)$ & $3(5.6)$ \\
NEs & 33 & $2(6.0)$ & $19(57.6)$ & $12(36.4)$ & $0(0.0)$ \\
\hline
\end{tabular}

Abbreviations: $\mathrm{HE} 4=$ human epididymis protein 4; $\mathrm{EC}=$ endometrial carcinoma; $\mathrm{NE}=$ normal endometria.
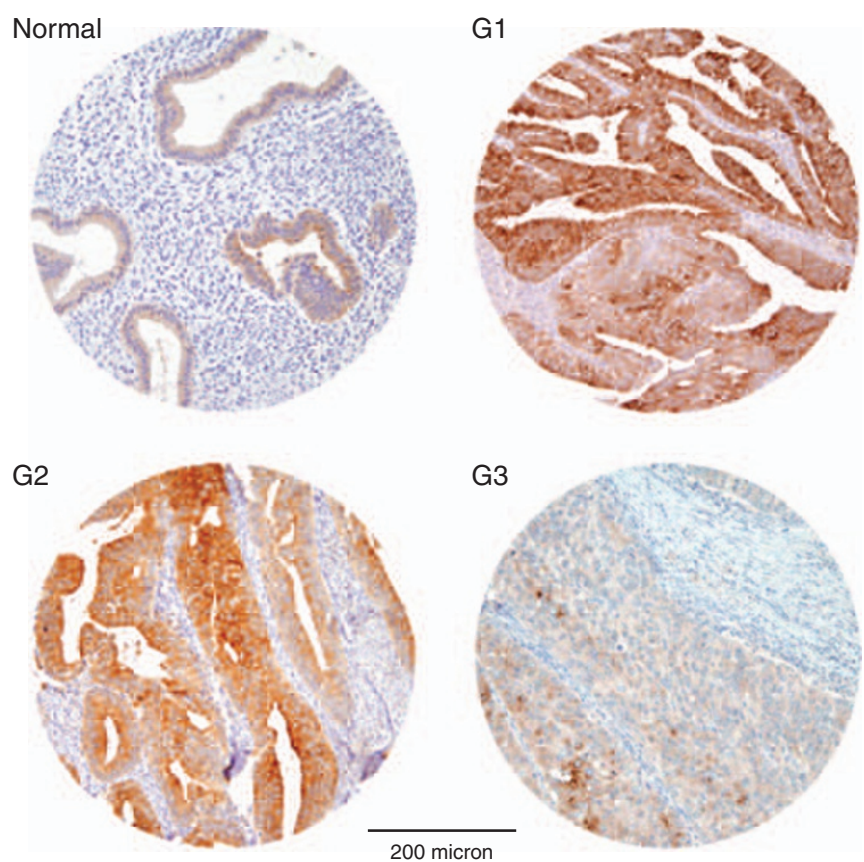

Figure 2 Representative immunohistochemical staining for HE4 in tissue microarrays of normal endometria (normal) and endometrial carcinomas (GI, G2 and G3). Normal tissues show predominantly a weak immunoreactivity for HE4 (mostly I+), whereas GI, G2 and G3 endometrial carcinomas are mainly scored $3+, 2+$ and $0 / 1+$, respectively. Magnification: × 100; scale bar length: 200 micron. 
A

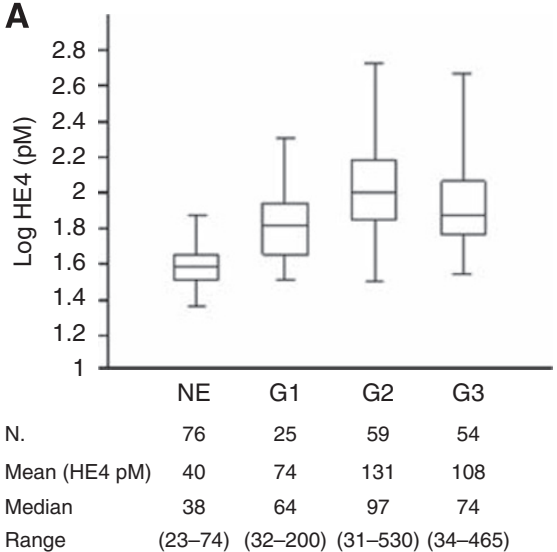

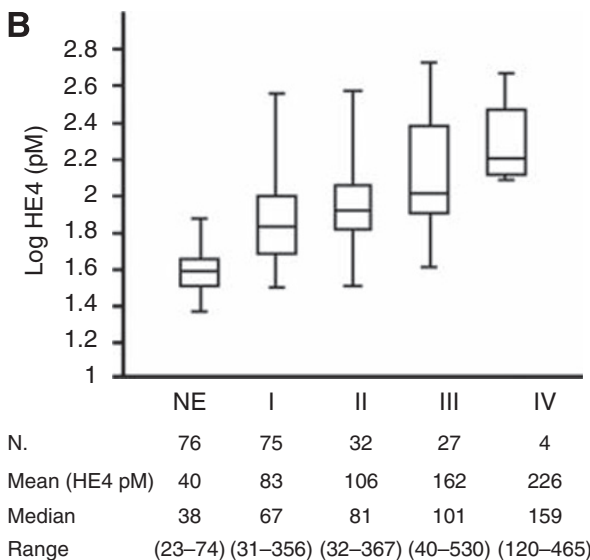

Figure 3 Box plots showing serum HE4 (sHE4) levels in controls with normal endometrium (NE) and in endometrial cancer patients, represented according to differentiation grade $(\mathbf{A})$ and to FIGO stage $(\mathbf{B})$

Table 2 Tumour biomarker sensitivities among patients with endometrial cancer

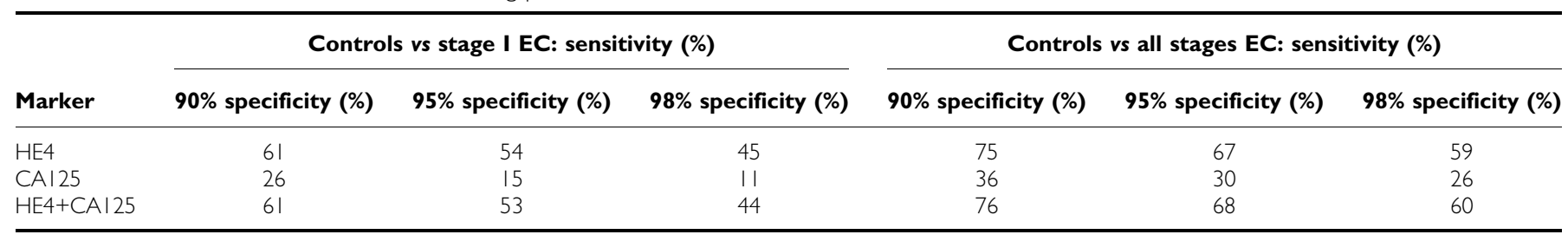

Abbreviations: CAI25 = cancer antigen 125; EC = endometrial carcinoma; HE4=human epididymis protein 4.

\section{Comparison between HE4 and CA125 serum levels and combination of the two markers}

We analysed CA125 serum levels in 127 EC patients and in $71 \mathrm{NEs}$, all tested with HE4 ELISA. Analysed CA125 median values were $11 \mathrm{U}$ (range 4-42), $12 \mathrm{U}$ (range 3-40), $20 \mathrm{U}$ (range 1-202) and $19 \mathrm{U}$ (range 4-853) for NEs, G1, G2 and G3 ECs, respectively. Median values of CA125 were $15 \mathrm{U}$ (range 1-114), $15 \mathrm{U}$ (range 4-50), $39 \mathrm{U}$ (range 4-202) and $67 \mathrm{U}$ (range 18-853) for stage I, stage II, stage III and stage IV ECs, respectively. The difference between serum CA125 levels in NEs compared with all ECs was statistically significant (median $\mathrm{ECs}=18 \mathrm{U}$, median $\mathrm{NEs}=11 \mathrm{U}$, $P<0.0001)$, whereas it was not significant when comparing NEs with G1 ECs $(P=0.99)$, NEs with stage I ECs $(P=0.95)$ and NEs with stage II ECs $(P=0.93)$. Logistic models were used to compare the sensitivity of CA125 and HE4 markers for the differentiation of ECs $v s$ NEs at set specificities of approximately 90,95 and $98 \%$ (Table 2). HE4 showed a considerably higher sensitivity compared with CA125 for detecting EC, considering all stages and all set specificities. For instance, HE4 had a sensitivity of $67 \%$ at a specificity of $95 \%$ compared with $30 \%$ for CA125. For stage I cancers, HE4 exhibited a $39 \%$ improvement in sensitivity at a specificity of $95 \%$ compared with CA125 alone (Table 2). As CA125 and HE4 serum levels were significantly correlated, but exhibited a low Spearman's rank coefficient $(P<0.01, \mathrm{rs}=0.38)$, a combination of the two markers was analysed. The combination of CA125 and HE4 led to a further improvement in sensitivity, even if limited, compared with HE4 alone, considering all set specificities and all EC stages. Examining only stage I EC patients, there was no gain in sensitivity when CA125 and HE4 were combined compared with HE4 alone.

\section{sHE4 levels and clinicopathological variables}

The relationship between sHE4 levels and the clinicopathological features of the 138 EC patients is shown in Table 3. Higher sHE4 levels were significantly associated with advanced age at diagnosis, menopause, higher FIGO stage and grade, deeper myometrial invasion, positive lymph nodes, presence of lymphovascular invasion, cervical and adnexal involvement, positive peritoneal cytology and administration of either chemotherapy or adjuvant radiation therapy.

\section{sHE4 levels and patient survival}

As expected, known EC clinical prognostic factors such as FIGO stage, histological type and lymph node involvement showed a statistically significant association with OS, PFS and DFS in univariate analyses (all $P<0.05$, data not shown), proving the validity of the patient cohort recruited in this study. In addition, as displayed in Figures 4A and B, respectively, higher HE4 serum levels (high $v s$ low HE4 tertiles) showed a significant association with poor OS $(P=0.02)$ and shorter PFS $(P=0.03)$. Regarding DFS (Figure 4C), medium vs low $\mathrm{HE} 4$ tertiles was significantly correlated with decreased DFS $(P=0.04)$, whereas the difference between high and low HE4 tertiles showed a marginal significance $(P=0.06)$. FIGO stage, histological type, lymph node involvement and HE4 serum levels were then included in a multivariate analysis. Non-endometrioid EC histological subtype, along with advanced FIGO stage, were identified as independent predictive factors for poor OS $(P=0.01$ and $P=0.04$, respectively, Table $4 \mathrm{~A})$, whereas sHE4 levels (medium vs low tertile) were shown to be marginally significant as prognostic factor for shorter OS $(P=0.08$, Table 4A). Regarding PFS, only histological type and, marginally, FIGO stages were of prognostic significance, whereas sHE4 levels were not (Table 4A). Regarding DFS, neither clinical parameters nor sHE4 levels were indicative of disease recurrence, even if elevated sHE4 levels exhibited the highest trend toward significance $(P=0.14$ and $P=0.17$, Table $4 \mathrm{~A})$. Then we performed a further survival analysis in the subgroup of 54 patients harbouring poorly differentiated ECs. The univariate model revealed that patients with elevated sHE4 levels (high tertile) had a significant poorer OS $(P=0.02$, Figure $4 \mathrm{D})$, shorter PFS $(P=0.02$, Figure $4 \mathrm{E})$ and worse DFS $(P=0.01$, Figure $4 \mathrm{~F})$ than patients with reduced 
Table 3 Clinical and pathological characteristics of 138 endometrial cancer patients and their association to HE4 serum levels (pM)

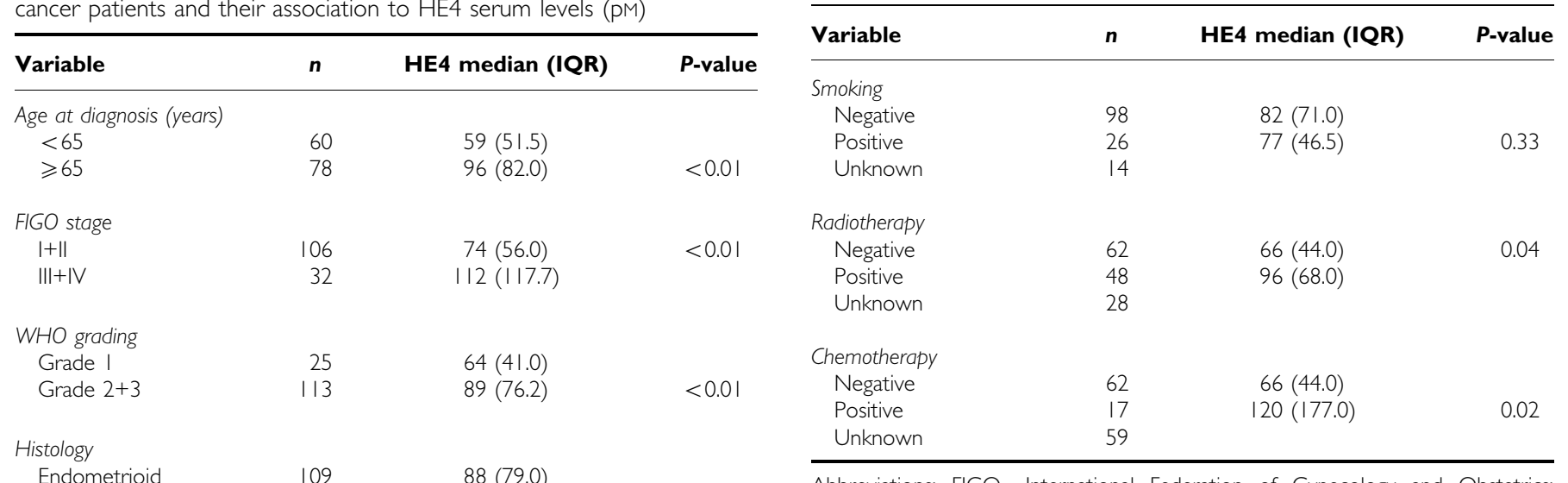

$\begin{array}{lll}\text { Endometrioid } & 109 & 88(79.0)\end{array}$

Non-endometrioid $\quad 29 \quad 70(522)$

Myometrial invasion $\mathrm{MO}+\mathrm{MI}$

$\mathrm{M} 2$

63

75

$66(42.5)$

$98(93.5)$

Lymph node status

Negative

Positive

Unknown

93

21

24

Cervical involvement

Absent

Stromal involvement

Unknown

Adnexal involvement

Negative

Positive

Peritoneal cytology

Negative

Positive

Unknown

Lymphovascular invasion

Absent

Present

Unknown

Parity

Nulliparity

Pluriparity

Unknown

Body mass index

$<25$

$\geqslant 25$

Unknown

Hypertension

Negative

Positive

Unknown

Diabetes

Negative

Positive

Unknown

\section{Menopause}

Negative

Positive

Unknown
Table 3 (Continued)

Abbreviations: $\mathrm{FIGO}=$ International Federation of Gynecology and Obstetrics; HE4 = human epididymis protein 4; $\mathrm{QR}=$ interquartile range; $\mathrm{WHO}=$ World Health Organisation.

sHE4 levels (low tertile). In multivariate analysis, sHE4 levels retained its significance as an independent prognostic factor for poor OS $(P=0.04)$, shorter PFS $(P=0.04)$ and decreased DFS $(P=0.01)$ in the subgroup of patients with poorly differentiated ECs (Table 4B).

\section{DISCUSSION} with favourable prognosis, because of the fact that the majority of patients declare their disease early by postmenopausal bleeding and therefore can be diagnosed at the first stage. However, an accurate serum marker for screening and early diagnosis would certainly be useful for those patients that may experience an increased risk of developing EC, such as those with severe obesity and diabetes, Lynch syndrome, PTEN gene defects or breast cancer women on Tamoxifen. Moreover, several high-risk EC groups, such as women with stages III-IV disease or even stage I patients harbouring high-grade carcinomas, or deep myometrial invasion, would benefit from a marker to give pre-operative indications, to monitor the effects of adjuvant therapy and to predict early tumour recurrence. Serum levels of CA125 are commonly used in the clinic for these purposes, but exhibit a low sensitivity and specificity (Sood et al, 1997; Powell et al, 2005). In the present investigation, with the aim to fully characterise human epididymis protein HE4 as a marker for EC, we have analysed its gene and protein expression in tumour tissues, its secretion in the sera and, finally, its prognostic value on a cohort of well-characterised EC patients. In a previous microarray study, Huhtinen et al (2009) examined HE4 gene expression in a limited number of EC samples, finding no significant difference with NEs. Conversely, according to our gene expression results, HE4 mRNA was significantly upregulated in ECs compared with NEs. Moreover, we demonstrated a significantly stronger HE4 immunohistochemical staining in EC compared with NE tissues. HE4 immunostaining in NE predominantly exhibits a weak score, whereas most EC samples show moderate/strong scores. Those results are in agreement with two previous reports regarding HE4 immunohistochemistry performed on a limited number of EC and NE samples (Drapkin et al, 2005; Galgano et al, 2006). Few recent studies have proposed HE4, a candidate molecular marker for ovarian cancer, as a promising serum marker for endometrial malignancies (Moore et al, 2008; Huhtinen et al, 2009; Montagnana et al, 2009). Serum CA125 and HE4 median values in EC and NE patients were shown to be similar in our investigation compared with previous studies, thus validating our patient cohort. In agreement with
$<0.01$ Endometrial carcinoma (EC) is generally considered a malignancy 
A Kaplan-Meier curves for OS

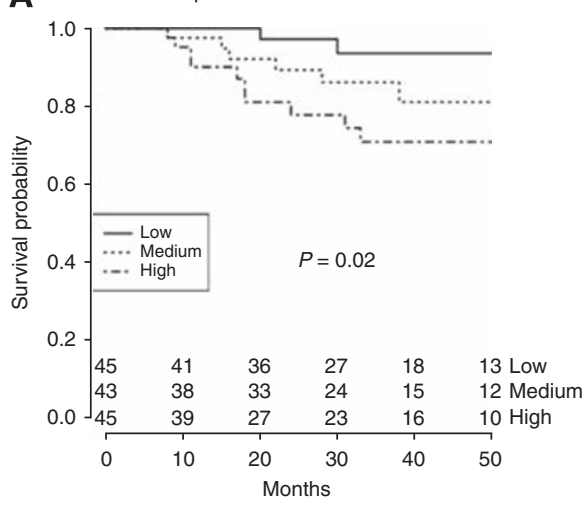

D

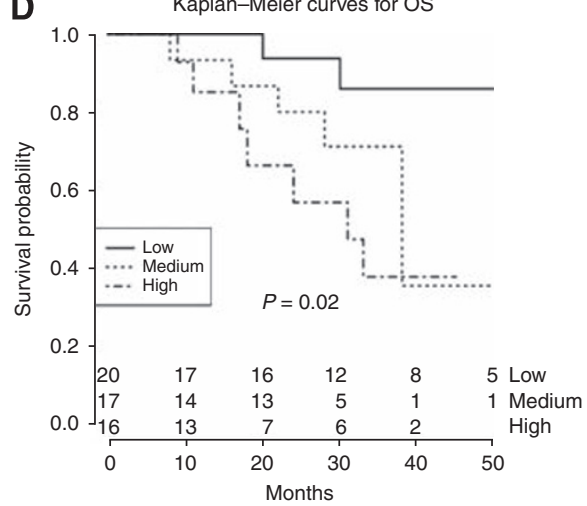

B

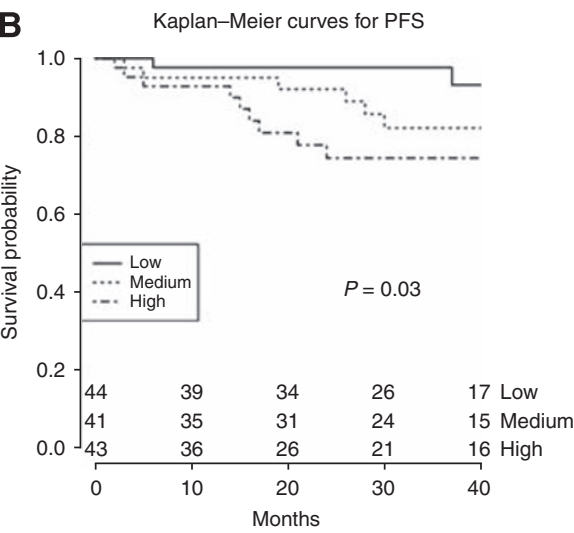

E

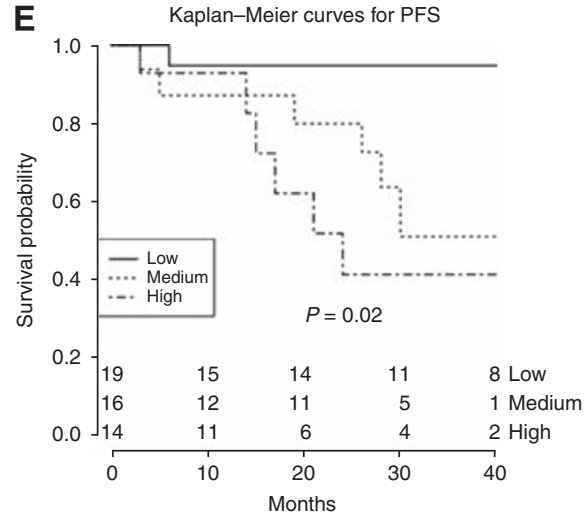

C Kaplan-Meier curves for DFS
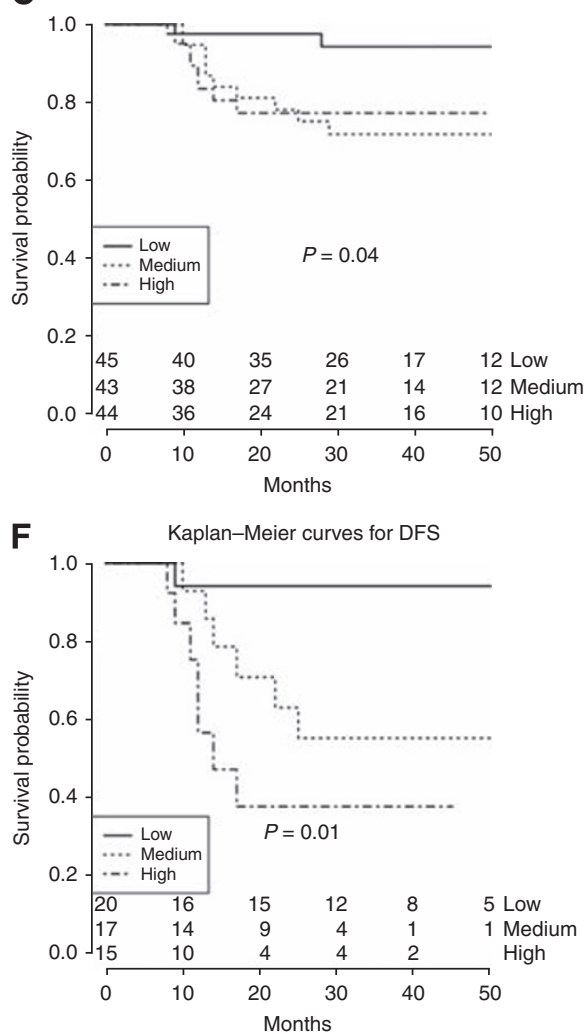

Figure 4 Kaplan-Meier survival curves for EC patients according to sHE4 levels on the entire patient cohort (A, overall survival; $\mathbf{B}$, progression-free survival; C, disease-free survival) and on poorly differentiated subgroup of EC patients (D, overall survival; E, progression-free survival; F, disease-free survival). P-values refer to the comparison between high vs low tertile, except for $\mathbf{C}$, in which medium vs low tertile shows to be significant.

Moore et al (2008) we observed significantly higher sHE4 levels in EC patients when compared with healthy women. Considering all EC stages, sHE4 sensitivity resulted to be higher than that of sCA125 in detecting cancer patients and, more importantly, we obtain the same result when sHE4 is tested for the detection of stage I EC. Interestingly, our study showed increased HE4 sensitivities at every specificities compared with those reported in the literature (Moore et al, 2008), regardless of the EC stage. Finally, the addition of HE4 to CA125 significantly raised the sensitivity compared with CA125 alone, either in the entire EC cohort (68 vs 30\% at 95\% specificity, respectively), or in stage I ECs (53 vs $15 \%$ at $95 \%$ specificity, respectively). Immunohistochemical staining data and sHE4 levels showed a low concordance coefficient in paired tumour samples. This discrepancy may be partially explained by the IHC semiquantitative scoring system and by the probable heterogeneity of the tumour tissue. Furthermore, this finding suggests that HE4 ELISA, that is a dynamic test to be performed at any time and offers precise marker quantification in serum, should be preferred to tissue immunostaining in EC clinical setting.

The HE4 higher sensitivity over CA125 in identifying stage I ECs indicates its potential prognostic value in detecting early tumour recurrence. There are limited evidence reporting sHE4 as a predictive marker for recurrence in ovarian cancer (Havrilesky et al, 2008; Anastasi et al, 2010), whereas to our knowledge, so far no prognostic value of sHE4 levels in EC patients has been investigated. Herein, for the first time we demonstrated the significant association between elevated sHE4 levels and adverse EC factors, which may suggest a relation between increased tumour biological aggressiveness and HE4 release in EC. Moreover, our study is the first to correlate sHE4 levels with clinical outcome in patients with EC. In univariate analysis on the entire EC cohort, we found that higher sHE4 levels were significantly more often observed in patients with poor OS, PFS and DFS. The multivariate analysis showed histological type and staging as independent prognostic factor for OS, whereas sHE4 levels did not reach the statistical significance. We then decided to focus our survival analysis on a high-risk subgroup of EC patients, harbouring poorly differentiated ECs, characterised by highly malignant cancers with poor prognosis (Bokhman, 1983). Remarkably, this is the first investigation reporting higher sHE4 levels as the only independent prognostic factor for shortened PFS and DFS in this selected group of patients. Regarding OS, sHE4 levels and histological types were independent prognostic factors, with the former showing a higher hazard ratio compared with the latter. Given these findings and the poor efficacy of current treatment modalities, it seems that patients with poorly differentiated ECs and high sHE4 levels could be managed more aggressively, using contemporary therapeutic options, than those with low sHE4 levels. However, these data should be confirmed with additional studies on larger patient cohort before routine sHE4 levels evaluation could be applied in the clinical setting.

Summarising, our results confirm that HE4 is an accurate and sensitive serum marker for early detection of EC patients, exhibiting a better diagnostic performance compared with CA125, which is the marker conventionally used in EC management. In addition, we demonstrated for the first time that high sHE4 levels may identify patients harbouring a more aggressive EC phenotype and may be an independent prognostic factor for OS, PFS and DFS in poorly differentiated EC patients. Therefore, the evaluation of sHE4 levels might be useful as an early, simple and highly efficient tool to select high-risk EC patients who could benefit from a tailored surgical (i.e., the extension of the lymph nodal dissection) and adjuvant (i.e., the extension of radiotherapy fields, systemic chemotherapy or both) therapy. Large prospective clinical studies 
Table 4 Multivariate analyses of OS, DFS and PFS in relation to clinical parameters and HE4 serum levels (A, entire cohort of EC patients; B, poorly differentiated EC patients)

\begin{tabular}{|c|c|c|c|c|c|c|c|c|c|}
\hline \multirow[b]{2}{*}{ Variable } & \multicolumn{3}{|c|}{ os } & \multicolumn{3}{|c|}{ DFS } & \multicolumn{3}{|c|}{ PFS } \\
\hline & HR & $95 \% \mathrm{Cl}$ & $P$-value & HR & $95 \% \mathrm{Cl}$ & $P$-value & HR & $95 \% \mathrm{Cl}$ & P-value \\
\hline \multicolumn{10}{|l|}{ (A) } \\
\hline \multicolumn{10}{|l|}{ Lymph node involvement } \\
\hline Positive vs negative & 0.67 & $0.07-6.11$ & 0.72 & 1.20 & $0.14-10.26$ & 0.87 & 0.53 & $0.06-5.06$ & 0.58 \\
\hline \multicolumn{10}{|l|}{ Histological type } \\
\hline \multicolumn{10}{|l|}{ FIGO stage } \\
\hline III+IV vs I+II & 20.32 & $1.79-230.40$ & 0.04 & 3.84 & $0.44-33.47$ & 0.20 & 11.72 & $1.13-121.31$ & 0.09 \\
\hline \multicolumn{10}{|l|}{ HE4 serum levels (tertiles) } \\
\hline High vs low & 2.84 & $0.54-15.01$ & 0.22 & 2.80 & $0.65-12.15$ & 0.17 & 2.76 & $0.47-16.14$ & 0.26 \\
\hline Medium vs low & 5.35 & $0.80-35.67$ & 0.08 & 3.05 & $0.69-|3.5|$ & 0.14 & 3.18 & $0.53-19.17$ & 0.21 \\
\hline \multicolumn{10}{|l|}{ (B) } \\
\hline \multicolumn{10}{|l|}{ Histological type } \\
\hline Non-endometrioid vs endometrioid & 1.06 & $0.21-5.29$ & 0.04 & 0.75 & $0.16-3.55$ & 0.31 & 0.98 & $0.22-4.39$ & 0.07 \\
\hline \multicolumn{10}{|l|}{ FIGO stage } \\
\hline||$|+| V$ vs I+II & 14.38 & $1.13-183.28$ & 0.94 & 3.36 & $0.32-35.47$ & 0.71 & 10.06 & $0.8|-| 25.7 \mid$ & 0.98 \\
\hline \multicolumn{10}{|l|}{ HE4 serum levels (tertiles) } \\
\hline High vs low & 6.85 & $1.02-46.17$ & 0.04 & 17.00 & $1.89-153.24$ & 0.01 & 9.81 & $1.01-95.26$ & 0.04 \\
\hline Medium vs low & 4.98 & $0.80-31.06$ & 0.08 & 5.10 & $0.50-51.00$ & 0.17 & 6.74 & $0.72-62.54$ & 0.09 \\
\hline
\end{tabular}

Abbreviations: $\mathrm{Cl}$ = confidence interval; DFS = disease-free survival; $\mathrm{EC}=$ endometrial carcinoma; FIGO = International Federation of Gynecology and Obstetrics; HE4 = human epididymis protein 4; $\mathrm{HR}=$ hazards ratio; $\mathrm{OS}=$ overall survival; $\mathrm{PFS}=$ progression-free survival; $\mathrm{WHO}=$ World Health Organisation

are certainly necessary to support these findings and to assess the potential of HE4 as a new tool for preoperative evaluation and postoperative surveillance of EC patients.

\section{ACKNOWLEDGEMENTS}

We wish to thank Prof Piergiovanni Grigolato and Dr Carla Donzelli for their excellent support to the project. We are also grateful to Dr Germana Tognon for many helpful discussions and Dr Giuseppina Ruggeri for CA125 serum determinations. Finally, we wish to thank all the nurses working in the OR and in the Division of Obstetric and Gynecology, Spedali Civili di Brescia, Italy, in particular Mrs Margherita Franzoni, for the essential contribution in the collection of samples. Financial support: This investigation was supported by grants from the Nocivelli Foundation, Brescia, Italy, and by grants from the Istituto Superiore di Sanità (Progetto Oncoproteomica, Programma Italia-USA 'Farmacogenomica Oncologica', convenzione 527/B4/4), Rome, Italy.

\section{Conflict of interest}

The authors declare no conflict of interest.

\section{REFERENCES}

Amant F, Moerman P, Neven P, Timmerman D, Van Limbergen E, Vergote I (2005) Endometrial cancer. Lancet 366(9484): 491-505

Anastasi E, Marchei GG, Viggiani V, Gennarini G, Frati L, Reale MG (2010) HE4: a new potential early biomarker for the recurrence of ovarian cancer. Tumour Biol 31(2): 113-119

Bignotti E, Tassi RA, Calza S, Ravaggi A, Romani C, Rossi E, Falchetti M, Odicino FE, Pecorelli S, Santin AD (2006) Differential gene expression profiles between tumor biopsies and short-term primary cultures of ovarian serous carcinomas: identification of novel molecular biomarkers for early diagnosis and therapy. Gynecol Oncol 103(2): 405-416

Bingle L, Singleton V, Bingle CD (2002) The putative ovarian tumour marker gene HE4 (WFDC2), is expressed in normal tissues and undergoes complex alternative splicing to yield multiple protein isoforms. Oncogene 21(17): 2768-2773

Bokhman JV (1983) Two pathogenetic types of endometrial carcinoma. Gynecol Oncol 15: 10-17
Cirisano Jr FD, Robboy SJ, Dodge RK, Bentley RC, Krigman HR, Synan IS, Soper JT, Clarke-Pearson DL (1999) Epidemiologic and surgicopathologic findings of papillary serous and clear cell endometrial cancers when compared to endometrioid carcinoma. Gynecol Oncol 74(3): 385-394

Cirisano Jr FD, Robboy SJ, Dodge RK, Bentley RC, Krigman HR, Synan IS, Soper JT, Clarke-Pearson DL (2000) The outcome of stage I-II clinically and surgically staged papillary serous and clear cell endometrial cancers when compared with endometrioid carcinoma. Gynecol Oncol 77(1): 55-65

Creasman WT, Odicino F, Maisonneuve P, Beller U, Benedet JL, Heintz AP, Ngan HY, Sideri M, Pecorelli S (2001) Carcinoma of the corpus uteri. J Epidemiol Biostat 6(1): 47-86

DeLong ER, DeLong DM, Clarke-Pearson DL (1988) Comparing the areas under two or more correlated receiver operating characteristic curves: a nonparametric approach. Biometrics 44(3): 837-845

Drapkin R, von Horsten HH, Lin Y, Mok SC, Crum CP, Welch WR, Hecht JL (2005) Human epididymis protein 4 (HE4) is a secreted glycoprotein 
that is overexpressed by serous and endometrioid ovarian carcinomas. Cancer Res 65(6): 2162-2169

Duk JM, Aalders JG, Fleuren GJ, de Bruijn HW (1986) CA125: a useful marker in endometrial carcinoma. Am J Obstet Gynecol 155(5): 1097-1102

Galgano MT, Hampton GM, Frierson Jr HF (2006) Comprehensive analysis of HE4 expression in normal and malignant human tissues. Mod Pathol 19(6): $847-853$

Gemer O, Uriev L, Harkovsky T, Peled R, Ben-Dor D, Barak F, Segal S (2004) The significance of the degree of myometrial invasion in patients with stage IB endometrial cancer. Eur J Gynaecol Oncol 25(3): 336-338

Greven K, Winter K, Underhill K, Fontenesci J, Cooper J, Burke T (2006) Final analysis of RTOG 9708: adjuvant postoperative irradiation combined with cisplatin/paclitaxel chemotherapy following surgery for patients with highrisk endometrial cancer. Gynecol Oncol 103(1): 155-159

Havrilesky LJ, Whitehead CM, Rubatt JM, Cheek RL, Groelke J, He Q, Malinowski DP, Fischer TJ, Berchuck A (2008) Evaluation of biomarker panels for early stage ovarian cancer detection and monitoring for disease recurrence. Gynecol Oncol 110(3): 374-382

Huhtinen K, Suvitie P, Hiissa J, Junnila J, Huvila J, Kujari H, Setälä M, Härkki P, Jalkanen J, Fraser J, Mäkinen J, Auranen A, Poutanen M, Perheentupa A (2009) Serum HE4 concentration differentiates malignant ovarian tumours from ovarian endometriotic cysts. Br J Cancer 100(8): $1315-1319$

Kirchhoff C, Habben I, Ivell R, Krull N (1991) A major human epididymisspecific cDNA encodes a protein with sequence homology to extracellular proteinase inhibitors. Biol Reprod 45(2): 350-357

Kohler MF, Carney P, Dodge R, Soper JT, Clarke-Pearson DL, Marks JR, Berchuck A (1996) p53 overexpression in advanced-stage endometrial adenocarcinoma. Am J Obstet Gynecol 175(5): 1246-1252

Maxwell GL, Risinger JI, Alvarez AA, Barrett JC, Berchuck A (2001) Favorable survival associated with microsatellite instability in endometrioid endometrial cancers. Obstet Gynecol 97(3): 417-422
Montagnana M, Lippi G, Ruzzenente O, Bresciani V, Danese E, Scevarolli S, Salvagno GL, Giudici S, Franchi M, Guidi GC (2009) The utility of serum human epididymis protein 4 (HE4) in patients with a pelvic mass. J Clin Lab Anal 23(5): 331 - 335

Moore RG, Brown AK, Miller MC, Badgwell D, Lu Z, Allard WJ, Granai CO, Bast Jr RC, Lu K (2008) Utility of a novel serum tumor biomarker HE4 in patients with endometrioid adenocarcinoma of the uterus. Gynecol Oncol 110(2): 196-201

Morrison C, Zanagnolo V, Ramirez N, Cohn DE, Kelbick N, Copeland L, Maxwell GL, Fowler JM (2006) HER-2 is an independent prognostic factor in endometrial cancer: association with outcome in a large cohort of surgically staged patients. J Clin Oncol 24(15): 2376-2385

Powell JL, Hill KA, Shiro BC, Diehl SJ, Gajewski WH (2005) Preoperative serum CA125 levels in treating endometrial cancer. J Reprod Med 50: $585-590$

R Development Core Team (2010) R: A language and environment for statistical computing. R Foundation for Statistical Computing, Vienna, Austria. ISBN 3-900051-07-0, URL http://www.R-project.org

Randall ME, Filiaci VL, Muss H, Spirtos NM, Mannel RS, Fowler J, Thigpen JT, Benda JA, Gynecologic Oncology Group Study (2006) Randomized phase III trial of whole-abdominal irradiation versus doxorubicin and cisplatin chemotherapy in advanced endometrial carcinoma: a Gynecologic Oncology Group Study. J Clin Oncol 24(1): 36-44

Risinger JI, Hayes K, Maxwell GL, Carney ME, Dodge RK, Barrett JC, Berchuck A (1998) PTEN mutation in endometrial cancers is associated with favorable clinical and pathologic characteristics. Clin Cancer Res 4(12): $3005-3010$

Sherman ME (2000) Theories of endometrial carcinogenesis: a multidisciplinary approach. Mod Pathol 13(3): 295-308

Sood AK, Buller RE, Burger RA, Dawson JD, Sorosky JI, Berman M (1997) Value of preoperative CA125 level in the management of uterine cancer and prediction of clinical outcome. Obstet Gynecol 90: 441-447 